\title{
Trajectory Design of Solar Sail Spacecraft for Interplanetary Rendezvous Missions
}

\author{
By Shinji HOKAMOTO'), Koji SACHIMOTO'), and Koki FUJITA ${ }^{1)}$ \\ ${ }^{1)}$ Department of Aeronautics and Astronautics, Kyushu University, Fukuoka, Japan
}

(Received May 2nd, 2008)

\begin{abstract}
This paper deals with in-plane motion of a solar sail spacecraft to rendezvous with a planet. In the interplanetary rendezvous problem, the spacecraft's velocity must coincide with the orbital velocity of the planet when it reaches the planet's orbit. Thus, the spacecraft's radial and tangential velocities as well as its orbital radius are controlled by one control input, i.e. the spacecraft's pitch angle. In this paper, we propose a trajectory design method which can reduce the amount of computational iterations considerably. This is applied to a rendezvous mission to a planet in a circular orbit and is achieved by utilizing locally optimal control techniques. A hidden problem in the method is pointed out, and a countermeasure is proposed. Then, numerical results of the proposed method are shown and compared with the results obtained by a fully numerical iteration method. Finally, some mathematical properties of a sailcraft's governing equations are discussed in the framework of nonlinear control theory. We show analytically that a solar sail spacecraft can rendezvous with any planet in any elliptical orbit by using only pitch angle control.
\end{abstract}

Key Words: Solar Sail, Trajectory Design, Locally Optimal Control, Nonlinear Theory

\section{Nomenclature}

$a \quad$ : semi-major axis, or acceleration

e : eccentricity

f : drift-term

$L \quad$ : lightness number

p : semi latus rectum

$r \quad$ : heliocentric orbital radius

$v \quad$ : velocity

$\boldsymbol{x} \quad$ : states vector

$\alpha \quad$ : sail's pitch angle

$\mu \quad$ : Sun's gravitational constant

$\theta \quad$ : true anomaly

$\omega \quad$ : argument of pericenter

$\xi \quad$ : orbital phase

$[\boldsymbol{f}, \boldsymbol{g}]$ : Lie bracket

Subscripts

$r \quad$ : radial directional

$\xi \quad$ : tangential directional

\section{Introduction}

Improvements of materials and fabrication techniques for solar sails re-encourage the researches on solar sail propulsion for future space missions. A solar sail propulsion system consumes no propellant mass by utilizing the solar radiation pressure forces. Furthermore, the solar radiation pressure forces are integrated during the whole mission, although the instantaneous propulsive force is small. Thus, the system is expected to show a large propulsive effect, Ft .

On the other hand, the sailcraft can not control the magnitude and direction of the acceleration vector independently. This is because the propulsion force is generated as the reaction force of solar photons impinging on the sail surface. Thus the magnitude and direction of the propulsion force are fixed according to the sailcraft's distance from and attitude angles to the sun. This restriction on the propulsion force distinguishes sailcraft trajectory design problems from the same problems for a spacecraft with control thrusters. Another distinctive problem of solar sail spacecraft is low rates of attitude motion due to its huge sail size.

Trajectory design problems of a solar sail spacecraft for interplanetary missions are highly nonlinear and have no closed-form solutions. Thus, most of previous studies for such problems depend on numerical iteration approaches. For example, references 1)-3) utilize numerical techniques based on optimal control techniques. However, numerical iteration approaches necessitate considerable amount of computations to get an acceptable solution. Furthermore, any revision of the conditions and model parameters usually requires another complete numerical iteration process, even when the revision is very small. In the numerical approach, an initial solution must be carefully selected, since it strongly influences the finally obtained solution. Thus, some studies apply "Genetic Algorithm" or "Evolutional Algorithm” to the search of initial solutions (for example, Dachwald ${ }^{4)}$ ).

This paper discusses solar sail's trajectory design problems for interplanetary rendezvous missions. In rendezvous missions, the sailcraft velocity must coincide with the planet's orbital velocity when it reaches the planet. To simplify the problem, this paper assumes that the starting and target planets are in circular orbits, and deals with in-plane motion of a solar sail spacecraft. First, the governing equations for orbital motion are represented. Then, a trajectory design method which can considerably reduce the computational iterations is 
proposed based on locally optimal control techniques. Finally, some mathematical properties of the sailcraft's governing equations are discussed in the framework of nonlinear control theory. Consequently, it is shown analytically that a solar sail spacecraft can rendezvous with any planet in any elliptical orbit by using only its pitch angle control.

\section{Orbital Motion}

To simplify the problem, we assume that only the sun's gravitational force and its solar radiation pressure force define the sailcraft's orbital motion. Therefore, no other disturbance forces are considered. The solar radiation pressure force is transferred into the sailcraft's propulsion force due to the solar photons impinging and reflecting on the sail structure.

If the reflection is assumed to be "perfect", the acceleration of the sailcraft is expressed in the radial and tangential directions as follows ${ }^{5}$.

$$
a_{r}=L \frac{\mu}{r^{2}} \cos ^{3} \alpha, a_{\xi}=L \frac{\mu}{r^{2}} \cos ^{2} \alpha \sin \alpha
$$

The ratio of the radiation force on the sail relative to the gravitational force defines the lightness number $L$. The $\alpha$ is the angle between the sail outward normal and the radial direction, and is the only control input of the sailcraft (Fig. 1).

Under the acceleration expressed in Eq. (1), the equations of motion for the sailcraft are expressed in a heliocentric inertial frame as follows.

$$
\begin{gathered}
\dot{r}=v_{r} \\
\dot{\xi}=\frac{v_{\xi}}{r} \\
\dot{v}_{r}=\frac{v_{\xi}^{2}}{r}-\frac{\mu}{r^{2}}+a_{r} \\
\dot{v}_{\xi}=-\frac{v_{r} v_{\xi}}{r}+a_{\xi}
\end{gathered}
$$

These are the expressions in a polar coordinate system (see Figs. 1 and 2).

Since the sailcraft is subjected to the solar radiation pressure force, its elliptic orbit changes over time. The perturbed orbits are called osculating orbits ${ }^{6)}$. Thus, Eqs. (2)-(5) can also be expressed in planar orbital elements as follows.

$$
\begin{gathered}
\dot{a}=\frac{2 a^{2}}{\sqrt{\mu p}}\left(a_{r} e \sin \theta+a_{\xi} \frac{p}{r}\right) \\
\dot{e}=\sqrt{\frac{p}{\mu}}\left[a_{r} \sin \theta+a_{\xi}\left\{\cos \theta+\frac{r}{p}(e+\cos \theta)\right\}\right] \\
\dot{\omega}=\frac{1}{e} \sqrt{\frac{p}{\mu}}\left\{-a_{r} \cos \theta+a_{\xi}\left(1+\frac{r}{p}\right) \sin \theta\right\} \\
\dot{\theta}=\frac{\sqrt{\mu p}}{r^{2}}-\dot{\omega}
\end{gathered}
$$

Eqs. (2)-(5) or Eqs. (6)-(9) are the governing equations of the sailcraft's orbital motion.

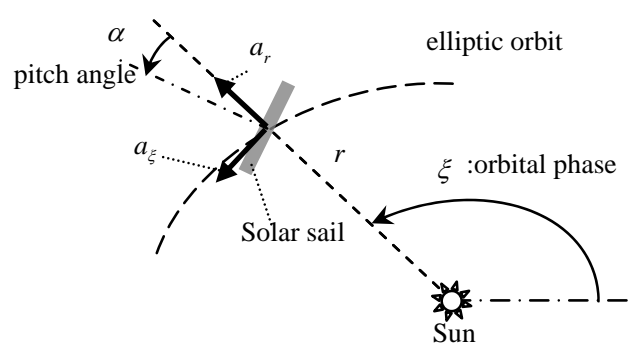

Fig. 1. Solar sail's pitch angle and its acceleration direction

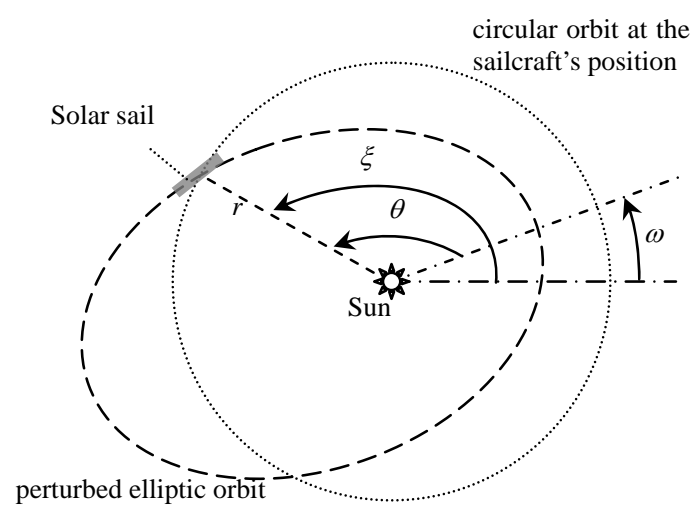

Fig. 2. Solar sail's orbital parameters

\section{Trajectory Design Method}

For a rendezvous problem to a planet, the solar sail's velocity must coincide with the planet orbital velocity when it reaches the planet. This implies that even for in-plane problem, four state variables - two for velocity and two for position must be controlled to their target values by only one input, the sail's pitch angle $\alpha$.

Most of previous studies for the interplanetary rendezvous problem use numerical iterations to search for solutions. Minimum-time problems are frequently formulated by considering the mission duration as a performance index for the trajectory design. However, the search process requires considerable computational iterations, because the system's governing equations are highly nonlinear, and because the number of state variables is much larger than the number of inputs.

This section first explains a method which substantially reduces the computational iterations by utilizing a locally optimality concept. Then, a hidden problem of the method is pointed out, and a countermeasure is proposed for this problem. Finally, numerical results of the proposed procedure are shown and compared with the results obtained by a fully numerical iteration method.

\subsection{Trajectory design based on local optimality}

To reduce the number of the control variables, we assume that the orbits of the departure and target planets are both circular. Furthermore, the orbital phase angle $\xi$ in Figs. 1 and 2 is adjusted to the target planet by designing a proper 
launch trajectory. That is, if the relative orbital angle between the two planets is "proper", the target planet is at the position that the sailcraft reaches in the orbit of the target planet.

The above assumption indicates that the governing equations of the system are Eqs. (2)(4)(5) in the polar coordinate system and Eqs. (6)-(8) in the osculating orbital elements. Moreover, the argument of pericenter $\omega$ loses significance as the orbital parameter at the initial and final states, because both orbits are circular. Therefore, the rendezvous problem considering here is accomplished when only two orbital parameters, i.e. semi-major axis $a$ and eccentricity $e$, are adjusted to the target ones.

For minimum-time control strategy, Eqs. (6) and (7) indicate that the "optimal" pitch angle to rapidly change its semi-major axis and eccentricity, respectively. Thus, combining the two time histories of the pitch angle can theoretically generate a minimum-time rendezvous trajectory to a target planet. A control method blending several locally optimal maneuvers is generally called Locally Optimal Control. The most frequently used blending technique is to take a weighted average of the strategy. However, there is no guideline for "proper" weighting functions, although the weighting functions strongly influence the final trajectories.

This paper takes a simple combination of minimum-time control strategy for the local optimality. First, the optimal pitch angle for the semi-major axis is used as long as possible from the initial position. In this period, the optimal pitch angle for the eccentricity is also calculated by a computer. Since the two optimal pitch angles are not consistent, they are continuously connected in a following transitional duration by taking account of the fastest rate of the solar sail pitch motion. After the duration, the optimal pitch angle for the eccentricity is adopted until the semi-major axis reaches the target value. Then the computer examines the error for the eccentricity at the final position. When the final eccentricity error is sufficiently close to zero, the pitch angle profile generates a rendezvous trajectory to the target planet. Consequently, the remaining parameter in the above trajectory design procedure is the time to start the middle transitional duration. Thus, the numerical iterations are substantially reduced in this procedure compared to the standard fully numerical searches. It should be noted that the calculated trajectory satisfies the rendezvous conditions, because adjusting both semi-major axis $a$ and eccentricity $e(=0)$ to their target values guarantees the orbital velocity of the target planet.

As another approach for locally optimal control, we can consider two solar sail spacecraft with the same physical parameters; one at the starting planet and the other at the target planet. The former sailcraft starts with the optimal pitch angle to change the semi-major axis, while the latter starts with an optimal angle for the eccentricity. If the two sailcraft can rendezvous somewhere midway, their trajectories form a rendezvous trajectory. However, except for the two initial orbits, the spacecraft's orbits are elliptical defined by four parameters: argument of pericenter $\omega$ and true anomaly $\theta$ as well as semi-major axis $a$ and eccentricity $e$. Thus, this approach is as difficult as the original rendezvous problem to adjust four orbital parameters to the target ones.

\subsection{Countermeasure for a locally optimal control}

The described locally optimal control technique frequently encounters a slow convergence rate of the eccentricity, although principally the eccentricity should decrease without the delay of convergence. In the slow convergence, the locally optimal control algorithm shifts the optimal pitch angle $\alpha$ to 90 deg after the eccentricity approaches to zero. Thus in this situation the solar radiation force as a propulsive force reduces to an extremely small level, and it makes the eccentricity convergence rate slow down.

This phenomenon happens through the following mechanism. Since $e=0$ is a singular point as seen in Eq. (8), the argument of pericenter $\omega$ begins to change very quickly according to $e \rightarrow 0$. Then the solar radiation pressure force is consumed for the rotation of the orbit holding the same eccentricity level. Thus the shape of the orbit is not corrected. As a result the true anomaly approaches to $90 \mathrm{deg}$, and the optimal pitch angle shifts to 90 deg.

Thus a countermeasure to avoid this situation is discarding the optimal pitch angle for the eccentricity after the eccentricity approaches to zero. As a simple countermeasure, a pitch angle to keep $d \omega / d t$ constant guarantees the effective eccentricity convergence

\subsection{Numerical results}

As a typical example, this paper considers a mission from Earth to Mars for a rendezvous trajectory design problem:

$$
\begin{array}{ll}
\text { Initial states: } & (a, e)=\left(r_{E}, 0\right) \\
\text { Final states: } & (a, e)=\left(r_{M}, 0\right)
\end{array}
$$

The argument of pericenter $\omega$ and the true anomaly $\theta$ of the initial and final orbits can not be specified, since the both orbits are circular. The lightness number of the solar sail spacecraft is specified $L=0.1021$ to compare the results of this paper with the results by Mengali and Quarta ${ }^{2}$.

Figure 3 shows the generated orbit when we take the locally optimal control technique with the explained countermeasure. The solid black line in each figure indicates the result for the proposed method. On the other hand, the gray line means the result of the fully-numerical-iteration method. Figure 3 (a) is the orbit from Earth to Mars. Figures 3 (b)-(e) depict the time histories of the variables in the polar coordinate system. Similarly, Figs. 3 (f)-(i) show the time histories of the osculating orbital parameters. Figure 4 compares the time histories of the proposed method to the fully-numericaliteration method. It should be noted that, once the pitch angle of the solar sail is decided on the Earth orbit, the initial values of $\omega$ and $\theta$ are specified.

The proposed procedure based on a locally optimal control technique is quite simple and does not require the numerical search for the initial profile of the pitch angle. Thus the procedure can show the results in just several seconds. Although the profile of the pitch angle in Fig. 4 shows a little difference from the optimal input, the profiles of the orbital parameters are quite similar to the optimal results. The resulting mission time of the proposed method is about 509 days, and the increase of the mission duration is only about 9 days compared with the fully-numerical-iteration method.

As another example, the mission achievement time to 


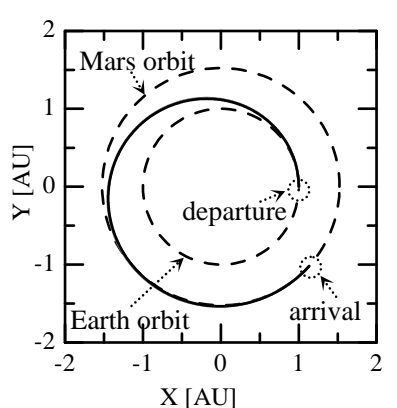

(a) The calculated orbits.

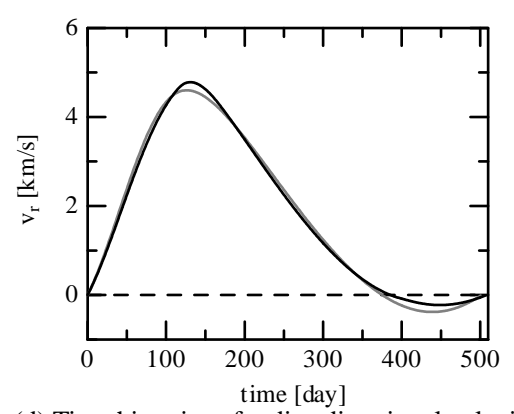

(d) Time histories of radius directional velocity

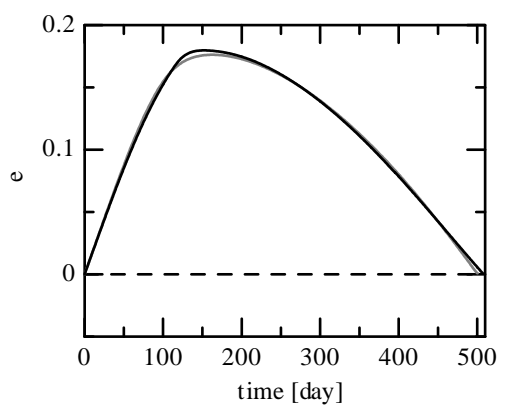

(g) Time histories of eccentricity

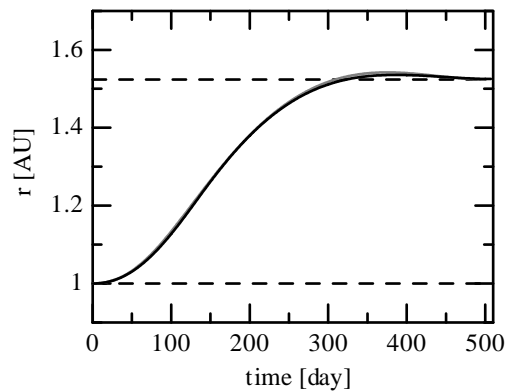

(b) Time histories of orbital radius

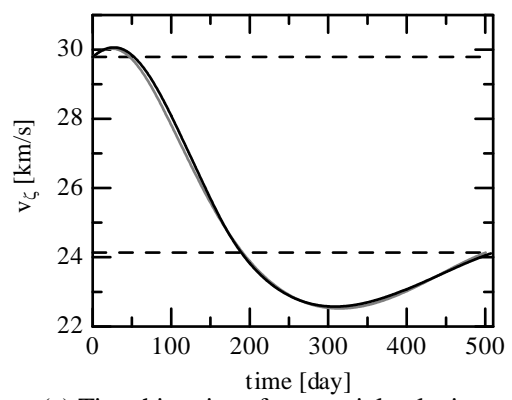

(e) Time histories of tangential velocity

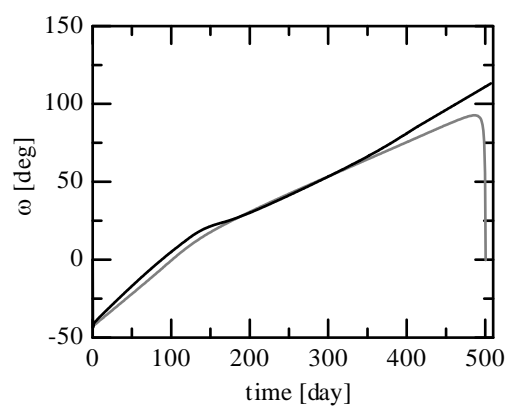

(h) Time histories of argument of pericenter

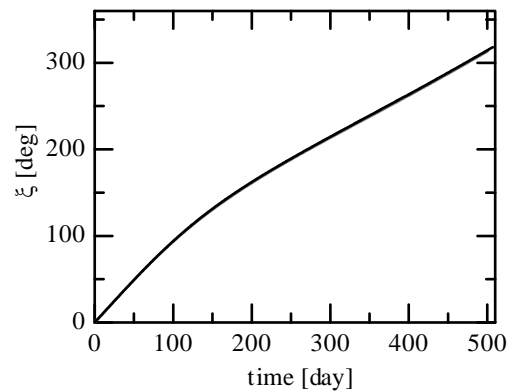

(c) Time histories of right ascension

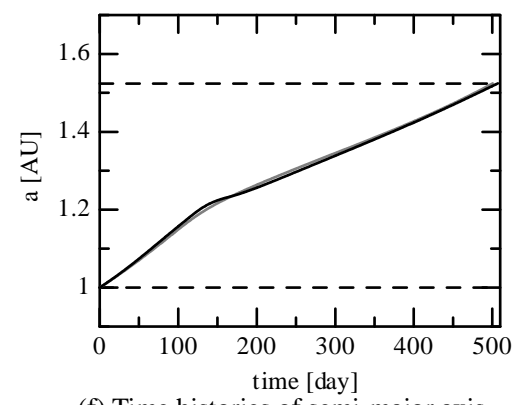

(f) Time histories of semi-major axis

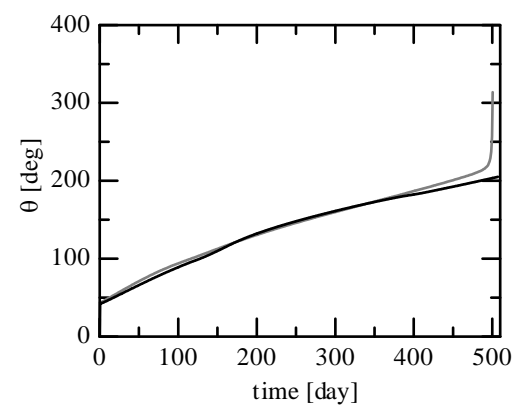

(i) Time histories of true anomaly

Fig. 3. Comparison of the orbital parameters ( $\_$proposed method, $\_$fully numerical method)

rendezvous with Mars has examined for different lightness numbers. Table 1 shows the results.

\section{Discussion as a Nonlinear System}

The trajectory design method based on locally optimal control techniques has several essential problems. One of those is that there is no guideline for blending the "optimal" inputs or weights. It should be noted that since the mission duration can be divided into more than two sections, a variety of blending methods must be examined in the trajectory design. Thus this method requires considerable amount of computational time for the "optimal" solution.

Another critical problem is the error at the final states. The governing equations of the solar sail spacecraft are highly nonlinear. Furthermore, the number of state variables to be controlled is much larger than the number of the control inputs. Therefore, controlling all the state variables exactly to the target ones by adjusting the switch-timing only once is impossible. This implies that the final states inevitably include some level of errors.
On the other hand, nonlinear theories ${ }^{7)}$ have been developed to analyze and control the state variables governed by nonlinear equations. Especially since late 1980's, dynamics and control for nonholonomic systems with non-integrable constraints, which is called "nonholonomic systems", have been attracting many researchers working in dynamics and control fields ${ }^{8)-11)}$. This is because, in a nonholonomic system, a number of generalized coordinates larger than its degrees of freedom may be controllable by appropriately designed time histories of control inputs.

One typical control strategy for nonholonomic systems utilizes both the original non-integrable constraints and the integrable constraints which emerge under some specific input patterns or in restricted areas of the states. In typical successful techniques, the integrable and non-integrable conditions are switched each other to control the states one by one. Thus integrability (or "involutiveness" of the states) of constraints is the most important concept for nonlinear control approaches. This section examines the integrability and accessibility of the governing equations of a sailcraft subjected to the solar radiation pressure. 


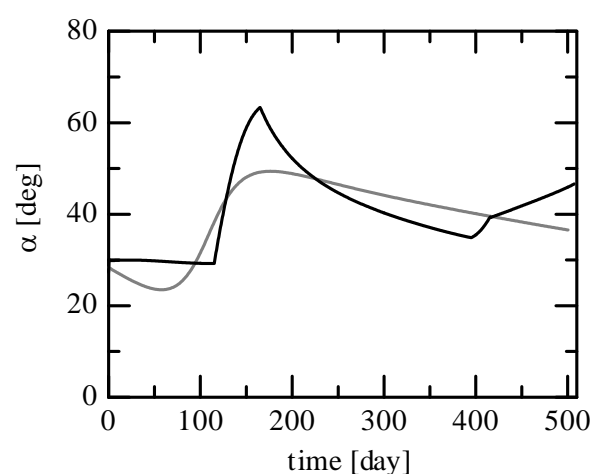

Fig. 4. Comparison of the pitch angles ( $\_$:proposed method, $\_$: fully numerical method)

A nonlinear system with a linear relationship with respect to input has been attracting many researchers in the last two decades. Such a nonlinear system is called an affine system and is expressed as follows.

$$
\dot{\boldsymbol{x}}(t)=\boldsymbol{f}(\boldsymbol{x})+\boldsymbol{g}(\boldsymbol{x}) \boldsymbol{u}(t)
$$

where $\boldsymbol{x}$ and $\boldsymbol{u}$ are its state and input vectors respectively, and the vector $\boldsymbol{f}$ denotes a drift-term. (An affine system without a drift-term is called a symmetrically affine system, and has been attracting special interest for its analytical treatment and control techniques.)

The solar sail's governing equations (2)-(5) are analyzed in the framework of nonlinear theories as below. (When we take Eqs. (6)-(9) as the governing equations, we can derive the same conclusions, but intermediate expressions become extremely complicate.)

$$
\boldsymbol{x}^{T}=\left[\begin{array}{llll}
r & \xi & v_{r} & v_{\xi}
\end{array}\right]
$$

is adopted here as the state vector. The input variable of the system is the pitch angle $\alpha$. However, the solar radiation pressure acting on the sailcraft is expressed in the cubed fashions of trigonometric functions, as seen in Eq. (1). This implies that Eqs. (2)-(5) are not categorized as an affine systems.

Hence, assuming that the pitch angle $\alpha$ is small, we consider an approximated subsystem. This idea is based on the following speculation. The original system can exhibit the same dynamics as the subsystem by restricting the input in small amplitudes, since the linearized input is a part of the nonlinear input. Therefore, if the state variables' motion of the subsystem is not restricted in a local constraint plane, the original nonlinear system's motion is also not restricted, that is "non-integrable". The accessibility concept of the original system can be discussed in the same manner.

The acceleration of the sailcraft can be expressed as follows for small pitch angle $\alpha$ :

$$
a_{r} \rightarrow L \frac{\mu}{r^{2}} \quad, \quad a_{\xi} \rightarrow L \frac{\mu}{r^{2}} \alpha
$$

Thus, the approximated subsystem can be described as an
Table 1. Mission achievement time for Mars rendezvous.

\begin{tabular}{|c|c|}
\hline Lightness number & Mission time [day] \\
\hline 0.109 & 498.5 \\
\hline 0.108 & 499.5 \\
\hline 0.107 & 501 \\
\hline 0.105 & 504 \\
\hline 0.1021 & 509 \\
\hline 0.100 & 513 \\
\hline 0.095 & 528.5 \\
\hline 0.900 & 553.5 \\
\hline 0.085 & 584.5 \\
\hline 0.084 & 589 \\
\hline
\end{tabular}

affine system:

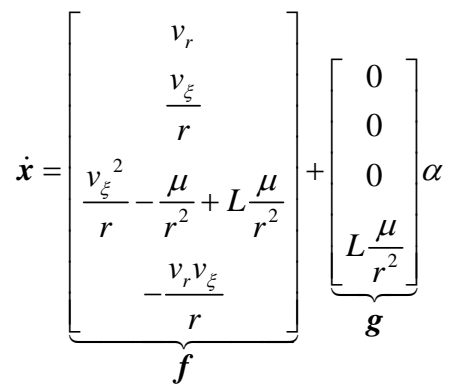

Lie bracket manipulation $[\boldsymbol{f}, \boldsymbol{g}]$ examines motion of the states relevant to the combined effect of the vectors $\boldsymbol{f}$ and $\boldsymbol{g}$. The drift-term $\boldsymbol{f}$ and the vector $\boldsymbol{g}$ in Eq. (13) indicate the following first-order Lie bracket.

$$
\begin{aligned}
{[\boldsymbol{f}, \boldsymbol{g}] } & =\frac{\partial \boldsymbol{g}}{\partial \boldsymbol{x}} \boldsymbol{f}-\frac{\partial \boldsymbol{f}}{\partial \boldsymbol{x}} \boldsymbol{g} \\
& =\left[\begin{array}{llll}
0 & -L \frac{\mu}{r^{3}} & -2 L \frac{\mu v_{\xi}}{r^{3}} & -L \frac{\mu v_{r}}{r^{3}}
\end{array}\right]^{T}
\end{aligned}
$$

In the same manipulation, the second-order Lie bracket $[\boldsymbol{g},[\boldsymbol{f}, \boldsymbol{g}]]$ is calculated as follows. (The other second-order Lie bracket $[\boldsymbol{f},[\boldsymbol{f}, \boldsymbol{g}]]$ shows a complicated expression.)

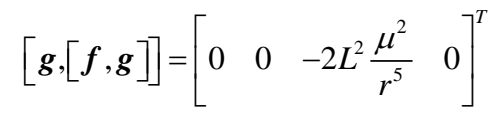

From Eqs. (13)-(15), the accessibility distribution of the approximated subsystem can be examined by using the following matrix:

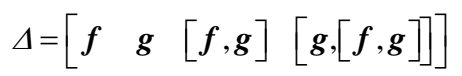

After some mathematical manipulations, the determinant of this matrix is arranged as follows.

$$
\operatorname{det}(\Delta)=\frac{2 L^{4} \mu^{4} v_{r}}{r^{10}}
$$


This expression indicates that $\operatorname{det}(\Delta)$ keeps non-zero value as long as $v_{r} \neq 0$. Since $v_{r}=0$ corresponds to a circular orbit, the matrix $\Delta$ has full rank in any point except its periapsis and apoapsis on any elliptical orbit. This indicates that the subsystem is accessible to any point in any elliptical orbit. Furthermore, the non-integrability of the subsystem is apparent, because the rank of the matrix $\Delta$ is beyond two.

From the above discussion, we can conclude that the solar sail's governing equations are non-integrable, and that at least the in-plane motion of the solar sail spacecraft can be transferred to any position in any elliptical orbit by using one control input $\alpha$.

\section{Conclusions}

This paper considers solar sail trajectory design problems for interplanetary rendezvous missions. First, we propose a trajectory design method which can considerably reduce computational iterations based on locally optimal control techniques. The procedure does not require the numerical search for the initial profile of the pitch angle, but the resulting orbit is quite similar to the one obtained by a fully-numerical-iteration method. Next, the governing equations of a solar sail's orbital motion are discussed in the framework of nonlinear control theory. It is shown analytically that a solar sail spacecraft can rendezvous with any planet in any elliptical orbit in the same orbital plane by using only the pitch angle control.

\section{References}

1) Colasurdo, G.. and Casalino, L.: Optimal Control Law for Interplanetary Trajectories with Nonideal Solar Sail, J. of Spacecraft and Rockets, 40 (2003), pp.260-265.

2) Mengali, G. and Quarta, A. A.: Optimal Three-Dimensional Interplanetary Rendezvous Using Nonideal Solar Sail, J. of Guidance, Control, and Dynamics, 28 (2005), pp.173-177.

3) McInnes, C. R, Mcdonald, M. and Dachwald, B.: Heliocentric Solar Sail Orbit Transfer with Locally Optimal Control Laws, J. of Spacecraft and Rockets, 44 (2007), pp.273-276.

4) Dachwald, B.: Optimal Solar-Sail Trajectories for Missions to the Outer Solar System, J. of Guidance, Control, and Dynamics, 28 (2005), pp.1187-1193.

5) McInnes, C. R: Solar Sailing - Technology, Dynamics and Mission Applications-, Springer-Praxis, Chichester, 1999, pp.32-55.

6) Vallado, D. A.: Fundamentals of Astrodynamics and Applications, Introduction to Fluid Dynamics, 2nd edition, Kluwer Academic Publishers, London, 2001, pp.575-593.

7) Isidori, A.: Nonlinear Control Systems, 2nd edition, Springer-Verlag, 1989.

8) Gurfil, P.: Control-Theoretic Analysis of Low-Thrust Orbital Transfer Using Orbital Elements, J. of Guidance, Control, and Dynamics, 26 (2003), pp.979-983.

9) Murray, R. M. and Sastry, S. S.: Nonholonomic Motion Planning: Steering Using Sinusoids, IEEE Transactions on Automatic Control, 38 (1993), pp.700-716.

10) Khenouf, H. and de Wit, C. C.: Quasi-Continuous Exponential Stabilizers for Nonholonomic Systems, Proceedings of 13th IFAC Triennial World Congress, (1996), pp.49-54.

11) Sordalen, O. J. and Egeland, O.: Exponential Stabilization of Nonholonomic Chained Systems, IEEE Transactions on Automatic Control, 40 (1995), pp.35-49. 